\title{
Multicolour photometry and Coravel observations of stars in the southern open cluster IC 2488 ${ }^{\star}, \star \star$
}

\author{
J. J. Clariá ${ }^{1}$, A. E. Piatti ${ }^{2}$, E. Lapasset ${ }^{1}$, and J.-C. Mermilliod ${ }^{3}$ \\ 1 Observatorio Astronómico, Universidad Nacional de Córdoba, Laprida 854, 5000 Córdoba, Argentina \\ e-mail: claria@mail .oac.uncor .edu; lapasset@mail.oac. uncor.edu \\ 2 Instituto de Astronomía y Física del Espacio, CC 67, Suc. 28, 1428 Buenos Aires, Argentina \\ e-mail: andres@iafe.uba.ar \\ 3 Institut d'Astronomie de l'Université de Lausanne, 1290 Chavannes-des-Bois, Switzerland \\ e-mail: Jean-Claude.Mermilliod@obs.unige.ch
}

Received 3 October 2002 / Accepted 10 December 2002

\begin{abstract}
We present new $U B V$ photoelectric observations of 119 stars in the field of the southern open cluster IC 2488, supplemented by $D D O$ and Washington photometry and Coravel radial velocities for a sample of red giant candidates. Nearly $50 \%$ of the stars sampled - including three red giants and one blue straggler - are found to be probable cluster members. Photometric membership probabilities of the red giant candidates show good agreement with those obtained from Coravel data. A mean radial velocity of $(-2.63 \pm 0.06) \mathrm{km} \mathrm{s}^{-1}$ is derived for the cluster giants. The reddening across the cluster is found to be uniform, the mean value being $E(B-V)=0.24 \pm 0.04$. IC 2488 , located at a distance of $(1250 \pm 120)$ pc from the Sun and $96 \mathrm{pc}$ below the Galactic plane, is most probably not related to the planetary nebula ESO 166-PN21. A metal abundance $[\mathrm{Fe} / \mathrm{H}]=0.10 \pm 0.06$ relative to the Sun is determined from $D D O$ data of the red giant members, in good agreement with the $[\mathrm{Fe} / \mathrm{H}]$ values derived from five independent Washington abundance indices. An age of $180 \mathrm{Myr}$ is determined from the fitting of isochrones computed with convective overshooting for $Z=0.019$. The isochrone for $\log t=8.25$ reproduces remarkably well not only the morphology of the upper main sequence but also the observed red giant pattern.
\end{abstract}

Key words. methods: observational - open clusters and associations: individual: IC2488 - star: abundances stars: Hertzsprung-Russell (HR) diagram and C-M diagrams

\section{Introduction}

The present study is part of a project currently being developed with the purpose of enhancing the quality of observationally determined properties for some southern relatively bright open clusters. To this effect, we have favoured the observation of clusters which were interesting not only because of the derivation of their fundamental parameters, but also because of the possibility of conducting a detailed analysis of the morphology of their giant evolutionary phases. We have used the $U B V, D D O$ and Washington photometric systems. The $D D O$ intermediate passband photometric system has been

Send offprint requests to: J. J. Clariá,

e-mail: claria@mail. oac. uncor.edu

* Based on observations made at Las Campanas Observatory (Chile) and Cerro Tololo Inter-American Observatory (Chile), National Optical Astronomy Observatories, operated by the Association of the Universities for Research in Astronomy, Inc., under contract with the National Science Foundation and on observations collected with the Danish 1.54-m telescope at the European Southern Observatory, La Silla (Chile).

$\star \star$ Tables 1 and $4 \mathrm{~b}$ are only available in electronic from at the CDS via anonymous ftp to cdsarc.u-strasbg.fr (130.79.128.5) or via

http://cdsweb.u-strasbg.fr/cgi-bin/qcat?J/A+A/399/543 specifically developed to be sensitive to the temperature, luminosity and metallicity of red giant stars, while the broad-band $U B V$ system has long been used to estimate distances, reddenings, and ages of star clusters. The Washington system also provides us with robust metal abundance estimates of red cluster giants through filters distributed over a wide wavelength baseline. Taking these reasons into consideration, we have searched in the literature for clusters with features indicative of the presence of giant clump candidates.

IC 2488 (C0926-567), located at $\alpha=9^{\mathrm{h}} 27^{\mathrm{m}} \cdot 6, \delta=-56^{\circ} 59^{\prime}$ (J2000) and $l=277^{\circ} .81, b=-4.40$, is an intermediate-richness, intermediate-brightness, detached cluster of Trumpler class II $2 \mathrm{~m}$ (Ruprecht 1966). To the best of our knowledge, this cluster has been previously studied photometrically only by Pedreros (1987, hereafter P87), who observed 32 stars in the cluster field in the $U B V$ system. He derived a colour excess $E(B-V)=0.26 \pm 0.02$, a distance $d=(1445 \pm 120) \mathrm{pc}$ and an age of $1.0 \times 10^{8} \mathrm{yr}$. More recently, IC 2488 has been the subject of proper motion studies by Baumgardt et al. (2000) and Dias et al. (2001), based on data drawn from the Hipparcos and Tycho2 Catalogues, respectively.

We have observed a number of stars in the cluster field nearly four times greater than that observed by P87, with the aim of discovering new members (especially A-type 
stars previously not identified by P87), which could define the colour-magnitude (CM) and colour-colour (CC) diagrams more accurately. A finding chart with our sequence of identification is shown in Fig. 1. We also present Coravel radialvelocity observations of 13 comparatively bright $\mathrm{G}$ and $\mathrm{K}$ stars located in the cluster field to discriminate between members and non members and detect spectroscopic binaries among the red giant candidates. In addition, as part of the project to determine abundances of red evolved stars in open clusters, DDO and Washingnton photometry have been obtained for 12 and 14 red giant candidates, respectively. These data are used to discuss the likelihood of membership, to determine individual $E(B-V)$ colour excesses, and to estimate independent metal abundance parameters. In Sect. 2 we describe the observational material and the data reduction. Section 3 presents the analysis and discussion of the $U B V$ data. Section 4 includes the analysis of the Coravel as well as the $D D O$ and Washington data of the red giant candidates, while the summary and final conclusions of the present work are drawn in Sect. 5.

\section{The observational material}

\subsection{Photometric data}

Most of the $U B V$ photometry reported here was obtained with the Cerro Tololo Inter-American Observatory (CTIO) 0.6-m and 1.0-m telescopes during four photometric nights in April 1994. Thirteen comparatively bright red stars in the cluster field had been previously measured in February 1986, with the University of Toronto's $0.6-\mathrm{m}$ telescope, located at Las Campanas Observatory (LCO). Single-channel photometers and pulse-counting electronics were used in both observatories. The CTIO measurements were made in conjunction with a dry-ice cooled Hamamatsu R943-02 GaAs photomultiplier, while those from LCO were performed with an EMI 9658 phototube. Mean extinction coefficients at both observatories were employed and nightly observations of about 12-17 standard stars, taken from the lists of Cousins (1973, 1974), Landolt (1973) and Graham (1982), were used to transform instrumental magnitudes into the $U B V$ standard system. The dispersions in the individual measures for the program stars yield the internal mean errors of a single $U B V$ observation which, of course, depend on the magnitude. These errors are $\sigma_{V} \leq 0.013 \mathrm{mag}$, $\sigma_{B-V} \leq 0.010 \mathrm{mag}$ and $\sigma_{U-B} \leq 0.017 \mathrm{mag}$ for $V<12 \mathrm{mag}$, increasing to $0.017,0.020$ and 0.028 for $V>12 \mathrm{mag}$, respectively. A comparison of the observed mean values with the published ones for the standard stars yields the external mean errors of a single observation, an indication of how closely the standard system has been reproduced. The average external mean errors are smaller than 0.015 mag for the $V$ magnitude and the $(B-V)$ and $(U-B)$ colours. All these errors are nearly similar to those in previous papers (e.g., Clariá et al. 1991).

Table 1 presents the results of our photometry for the stars numbered in Fig. 1. The first column gives a running star number. Columns (2) and (3) refer to linear coordinates $X$ and $Y$ in pixels for the positions of stars in Fig. 1. The figure is $1200 \times 1200$ pixels and the zero point corresponds to the upper left corner. Columns (4)-(7) give in succession the $V$ magnitude, the $B-V$ and $U-B$ colours and the number of
Table 1. $U B V$ photometry for stars in the field of IC 2488 .

\begin{tabular}{rcccccll}
\hline \hline No & $X$ & $Y$ & $V$ & $B-V$ & $U-B$ & $n$ & Remarks \\
\hline 1 & 1147 & 506 & 10.297 & 1.340 & 1.462 & 4 & P1, NM \\
2 & 1124 & 213 & 9.890 & 1.400 & 1.591 & 4 & NM \\
3 & 1155 & 590 & 12.639 & 0.296 & 0.223 & 1 & $\mathrm{M}$ \\
4 & 1127 & 565 & 12.782 & 0.693 & 0.187 & 1 & NM \\
5 & 1145 & 889 & 11.514 & 0.547 & 0.034 & 1 & NM \\
\hline
\end{tabular}

Note: Table 1 is available in their entirety in electronic form at the CDS. A portion of Table 1 is shown here for guidance regarding its form and content.

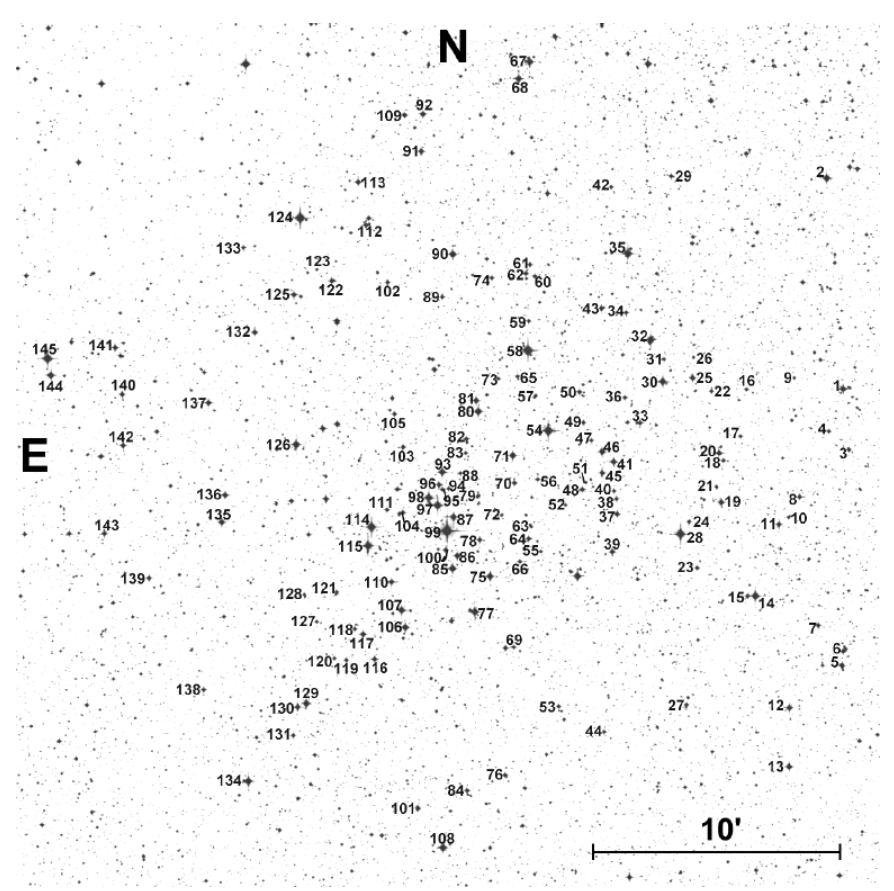

Fig. 1. Identification chart for stars observed in the field of IC 2488. The positions of the numbered stars can be determined from the linear coordinates $X$ and $Y$ listed in Table 1 . The zero point of these coordinates corresponds to the upper left corner and the size of the figure is $1200 \times 1200$ pixels.

photoelectric observations made on different nights. The last column of Table 1 provides the HD and/or CD numbers (when available) and an indication whether the star is believed to be a probable member $(\mathrm{M})$, a possible member (PM), or a nonmember (NM), according to the photometric criteria described in Sect. 3.1. Star numbers from P87 are preceded by P in this column, while confirmed red giant members (see Sect. 4.1) are indicated by RG. Table 1 is available in electronic form at the CDS via anonymous ftp to cdsarc.u-strasbg. fr. Adopted values for stars with more than one measurement are the unweighted means for individual observations. The values which appear between brackets in this table represent the magnitudes and colours only measured by P87. Only six stars were measured in common with P87, the mean differences ( $\Delta=$ previous minus present study) and standard deviations being: $\Delta V=$ $0.00 \pm 0.01, \Delta(B-V)=0.00 \pm 0.01$ and $\Delta(U-B)=-0.01 \pm$ 0.04 . Therefore, we decided to average the magnitudes and colours measured by ourselves and those measured by P87, for these six stars. 
Table 2. $D D O$ photometry of red giant candidates in IC 2488 .

\begin{tabular}{rccccccc}
\hline \hline No & $C 4548$ & $\sigma$ & $C 4245$ & $\sigma$ & $C 4142$ & $\sigma$ & $n_{2}$ \\
\hline 1 & 1.294 & 0.014 & 1.114 & 0.021 & 0.298 & 0.012 & 2 \\
2 & 1.315 & 0.012 & 1.156 & 0.012 & 0.277 & 0.017 & 2 \\
30 & 1.311 & 0.001 & 0.947 & 0.008 & 0.334 & 0.019 & 2 \\
35 & 1.225 & 0.015 & 0.949 & 0.009 & 0.203 & 0.014 & 5 \\
67 & 1.134 & 0.008 & 0.663 & 0.008 & 0.181 & 0.034 & 3 \\
68 & 1.384 & 0.004 & 1.243 & 0.010 & 0.305 & 0.002 & 3 \\
97 & 1.345 & 0.015 & 0.982 & 0.012 & 0.343 & 0.018 & 5 \\
115 & 1.402 & 0.016 & 1.111 & 0.018 & 0.409 & 0.019 & 5 \\
126 & 1.371 & 0.022 & 1.068 & 0.007 & 0.341 & 0.024 & 5 \\
129 & 1.368 & 0.002 & 1.212 & 0.011 & 0.266 & 0.004 & 3 \\
144 & 1.399 & 0.016 & 1.134 & 0.003 & 0.105 & 0.001 & 2 \\
145 & 1.258 & 0.021 & 1.004 & 0.025 & 0.292 & 0.022 & 4 \\
\hline
\end{tabular}

A total of fourteen stars in the cluster field brighter than $V=10.60$ and redder than $B-V=0.95$, were selected as red giant candidates from the CM diagrams (see Sect. 4.1). All these stars were observed in the Washington photometric system (Canterna 1976), while twelve of them were also measured in the $D D O$ system (McClure 1976), with a view to obtaining additional information about probable cluster membership, reddening and metallicity. Only the four primary filters of the $D D O(41,42,45$, and 48) and Washington $\left(C, M, T_{1}\right.$, and $\left.T_{2}\right)$ systems were used, since these provide enough adequate information for present purposes. The $D D O$ observations were carried out with the CTIO 1.0-m telescope in January 1991 and January 1992, while the $C M T_{1} T_{2}$ measurements were performed with the CTIO 0.6-m telescope in April 1994. All these observations employed a single-channel dry-ice cooled photometer and pulse-counting electronics. EMI 9781A and Hamamatsu R943-02 GaAs photomultipliers were utilized for the $D D O$ and $C M T_{1} T_{2}$ measurements, respectively. The transformation to the standard $D D O$ and Washington systems was accomplished by nightly observing between 12 and 17 standard stars from the lists of McClure (1976), Dean (1981), Canterna (1976) and Harris \& Canterna (1979). A few stars accurately measured several times by Clariá \& Lapasset (1985) in the field of the open cluster NGC 5822, were also used as Washington standard stars. The average of the rms errors from the transformation to the $D D O$ standard system of the different nights yielded the following values: $0.010,0.008$, and $0.010 \mathrm{mag}$ for $C(45-48), C(42-45)$ and $C(41-42)$, respectively. For the Washington photometry these values are $0.010,0.007,0.009$ and $0.019 \mathrm{mag}$ for $C-M, M-T_{1}$, $T_{1}-T_{2}$ and $T_{1}$, respectively. Tables 2 and 3 display the new $D D O$ and $C M T_{1} T_{2}$ data for probable red giants in the cluster field, along with the standard deviations of different individual observations, and the number of nights $n_{2}$ and $n_{3}$ on which each star was observed in the $D D O$ and Washington system, respectively.

\subsection{Radial-velocity observations}

Radial-velocity observations were obtained with the CORAVEL instrument (Baranne et al. 1979) at the 1.54-m
Danish telescope at ESO, La Silla, Chile. At least three observations were obtained through the period 1986-1994 for the radial velocity non-variable stars during the course of a long-term systematic program on cluster red giants conducted in Chile since 1983. The radial velocities are on the system defined by Udry et al. (1999) from high-precision radialvelocities obtained with the ELODIE spectrograph (Baranne et al. 1996). This calibration corrects for all systematic errors of the CORAVEL system.

Our mean results for individual stars in IC 2488 are summarized in Table 4 which gives the number in the present work, the mean radial velocity, probable error of the mean, both in $\left[\mathrm{km} \mathrm{s}^{-1}\right]$, the number of radial-velocity observations, the ratio of the external to internal errors $(E / I)$, the probability $P\left(\chi^{2}\right)$ that the scatter is due to random noise, the time interval in days covered by the observations of each star, and remarks on membership and duplicity. The individual observations are available only in electronic form from the Strasbourg service for published tables (http://cdsweb.u-strasbg.fr/cgi-bin/qcat?J/A+A/399/543).

\section{3. $U B V$ analysis}

\subsection{Membership criteria}

Figures 2, 3 and 4 show the observed CM and CC diagrams constructed from the photometric data in Table 1 . Note that 26 stars included in these diagrams have been measured only by P87 (see Table 1). For the sake of comparison, the Zero Age Main Sequence (ZAMS) of Girardi et al. (2000) is also shown with solid lines. Well-defined main sequences (MSs), typical of a moderately young open cluster, are clearly seen in the $U B V$ diagrams. There is, however, a substantial number of apparently foreground stars distributed over the cluster field. The MS consists of late-B up to mid-A type stars, extending over a range of nearly 3 mag. The width of the MS, if real, is probably due to the presence of unresolved binaries as well as to some amount of differential reddening rather than to observational scatter.

Except for the stars with $V<10.60$ and $B-V>0.95$ which will be discussed in Sect. 4, cluster members were selected only on condition that they fulfilled the following requirements: first, the location of a star in both CM diagrams had to correspond to the same evolutionary stage, and second, the location of the star in the CC diagram had to be close to the MS, the maximum departure accepted being $0.10 \mathrm{mag}$. Because several factors contribute to increase the scatter in the $U B V$ diagrams, a star located at most 0.75 mag above the cluster MS in Fig. 2, is considered to belong to it. Sixty three stars satisfy the above conditions while sixty nine stars meet only one or none of them. These stars are represented in the $U B V$ diagrams by filled and open circles, respectively. The positions of thirteen stars in Figs. 2, 3 and 4 preclude us from unambiguously retaining or discarding them as probable cluster members. These stars, represented by filled triangles, are thus considered to be possible members. The blue star \#99 (CD-56 2787), located near the cluster centre, is the brightest of the stars observed in the cluster field. As pointed out by P87, this is a probable 
Table 3. Washington photometry of red giant candidates in IC 2488.

\begin{tabular}{rccccccccc}
\hline \hline Star & $C-M$ & $\sigma$ & $M-T_{1}$ & $\sigma$ & $T_{1}-T_{2}$ & $\sigma$ & $T_{1}$ & $\sigma$ & $n_{3}$ \\
\hline 1 & 1.683 & 0.005 & 1.054 & 0.009 & 0.671 & 0.009 & 9.600 & 0.032 & 3 \\
2 & 1.760 & 0.008 & 1.088 & 0.008 & 0.701 & 0.006 & 9.162 & 0.026 & 3 \\
30 & 1.580 & 0.003 & 1.004 & 0.005 & 0.661 & 0.003 & 9.224 & 0.025 & 3 \\
35 & 1.387 & 0.004 & 0.891 & 0.004 & 0.585 & 0.008 & 9.821 & 0.024 & 3 \\
46 & 1.850 & 0.007 & 1.178 & 0.002 & 0.808 & 0.005 & 9.643 & 0.002 & 2 \\
67 & 1.035 & 0.004 & 0.869 & 0.009 & 0.612 & 0.011 & 9.565 & 0.026 & 2 \\
68 & 1.978 & 0.004 & 1.236 & 0.007 & 0.831 & 0.002 & 9.067 & 0.036 & 3 \\
97 & 1.684 & 0.009 & 1.052 & 0.005 & 0.697 & 0.005 & 8.716 & 0.029 & 3 \\
113 & 2.117 & 0.012 & 1.334 & 0.006 & 0.914 & 0.004 & 9.736 & 0.020 & 2 \\
115 & 1.879 & 0.003 & 1.158 & 0.006 & 0.762 & 0.005 & 8.423 & 0.006 & 3 \\
126 & 1.785 & 0.005 & 1.114 & 0.004 & 0.735 & 0.001 & 8.849 & 0.025 & 3 \\
129 & 1.890 & 0.003 & 1.198 & 0.006 & 0.791 & 0.007 & 9.254 & 0.029 & 3 \\
144 & 1.958 & 0.008 & 1.500 & 0.007 & 1.740 & 0.009 & 8.895 & 0.049 & 3 \\
145 & 1.539 & 0.003 & 0.954 & 0.009 & 0.621 & 0.003 & 8.705 & 0.031 & 3 \\
\hline
\end{tabular}

Table 4. Radial velocity of red giant candidates in IC 2488 .

\begin{tabular}{rrcrrrrl}
\hline \hline Star & $V_{\mathrm{r}}$ & $\epsilon$ & $N$ & $E / I$ & $P\left(\chi^{2}\right)$ & $\Delta T$ & Remarks \\
\hline 1 & 13.68 & 0.20 & 3 & 0.52 & 0.765 & 1846 & $\mathrm{NM}$ \\
2 & 29.96 & 0.19 & 3 & 0.72 & 0.599 & 1846 & $\mathrm{NM}$ \\
30 & 12.07 & 0.19 & 3 & 0.66 & 0.644 & 1846 & $\mathrm{NM}$ \\
35 & 7.04 & 0.18 & 3 & 0.17 & 0.971 & 1846 & $\mathrm{NM}$ \\
54 & 6.34 & 0.33 & 3 & 0.87 & 0.469 & 1846 & $\mathrm{NM}$ \\
67 & 14.26 & 0.12 & 26 & 28.75 & 0.000 & 3627 & $\mathrm{NM}, \mathrm{SB}$ \\
68 & 2.94 & 0.23 & 3 & 1.20 & 0.241 & 1118 & $\mathrm{NM}$ \\
97 & -2.48 & 0.52 & 5 & 3.57 & 0.000 & 3293 & $\mathrm{M}, \mathrm{SB}$ \\
115 & -2.70 & 0.16 & 4 & 0.60 & 0.779 & 2904 & $\mathrm{M}$ \\
126 & -2.71 & 0.17 & 4 & 0.47 & 0.882 & 2904 & $\mathrm{M}$ \\
129 & 31.16 & 0.20 & 3 & 0.62 & 0.684 & 1846 & $\mathrm{NM}$ \\
144 & 9.73 & 0.46 & 3 & 1.79 & 0.041 & 1846 & $\mathrm{NM}$ \\
145 & 5.11 & 0.17 & 3 & 0.75 & 0.572 & 1846 & $\mathrm{NM}$ \\
\hline
\end{tabular}

blue straggler deserving further study. The stars considered to be probable members are distributed within a circle of nearly 15 arcmin of radius, which has been adopted as the cluster radius.

Even though the general reliability of photometrically attributed membership is not called into question, we must not rule out the possibility of defective individual membership determinations. In particular, the use of the $U B V$ diagrams might have led us to discard some peculiar stars which are, in actual fact, cluster members. It is clear, nonetheless, that the exclusion of a few genuine members will have a virtually insignificant impact on the derivation of the fundamental cluster parameters.

Various kinematic studies have recently focussed on stars in the field of relatively bright open clusters with the aim of establishing membership probabilities and/or deriving mean cluster absolute proper motions. Based on photometric data, Glushkova et al. (1997) have determined absolute proper motions for 181 young open clusters, IC 2488 amongst them. From 8 stars in IC 2488, they obtained $\left\langle\mu_{\mathrm{RA}}\right\rangle=-4.6 \pm$

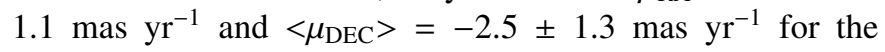

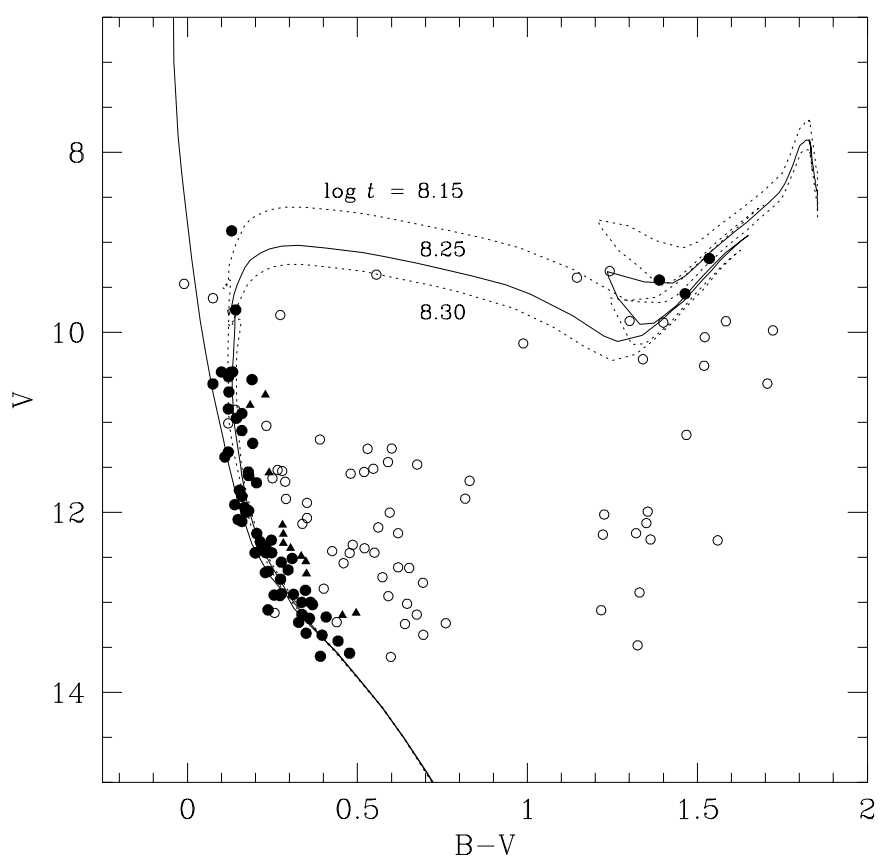

Fig. 2. The observed $[V, B-V]$ diagram for IC 2488. The ZAMS (solid line) and the theoretical isochrones computed by Girardi et al. (2000) for $Z=0.019$ and $\log t=8.15,8.25$ (solid line) and 8.30, have been adjusted to $E(B-V)=0.24$ and $V-M_{V}=11.20$. Filled and open circles represent probable members an non members, respectively, while the triangles indicate stars considered to be possible members.

proper motions in Right Ascension and Declination, respectively. Using data from the Hipparcos Catalogue, Baumgardt et al. (2000) determined mean proper motions for star \#99 (see

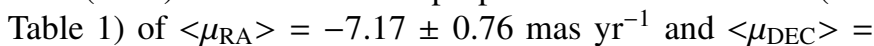
$6.47 \pm 0.73$ mas $\mathrm{yr}^{-1}$. On the other hand, from the Tycho2 Catalogue Dias et al. (2001) obtained mean cluster absolute

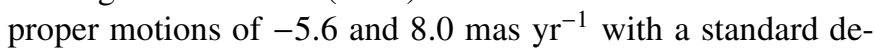

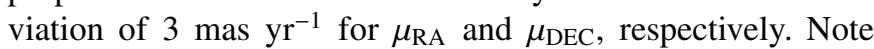
that the mean proper motions of Glushkova et al. seem to be in disagreement with those of Dias et al. and Baumgardt et al. In the subsequent analysis, we chose the study of 


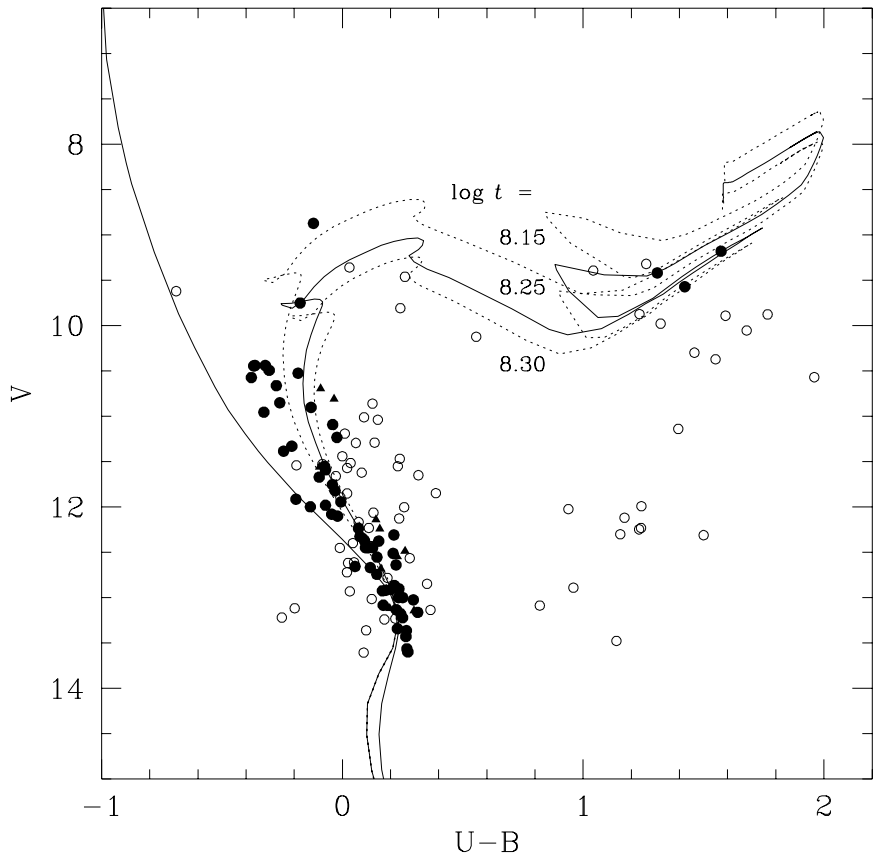

Fig. 3. The observed $[V, U-B]$ diagram for IC 2488 . The ZAMS (solid line) and the theoretical isochrones computed by Girardi et al. (2000) for $Z=0.019$ and $\log t=8.15,8.25$ (solid line) and 8.30, have been adjusted to $E(U-B)=0.17$ and $V-M_{V}=11.20$. Symbols are as in Fig. 2.

Dias et al. because it is based on a significantly larger number of stars than those used by Glushkova et al., with estimated membership probabilities. In addition, the latter values are in very good agreement with those derived for star \#99 by Baumgardt et al. (2000), which reinforces P87 and present photometric membership status of the star as a probable member (M). For the surrounding field, Dias et al.

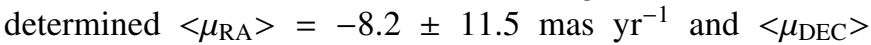
$=6.2 \pm 11.1$ mas $\mathrm{yr}^{-1}$. They used 40 and 23 stars respectively in order to estimate the mean proper motions of the cluster and its field, based on the membership probabilities of individual stars obtained from the proper motion data by applying the statistical method proposed by Sanders (1971). The last column of Table 1 includes their membership probabilities besides our present adopted membership status for 58 stars in common. $13 \mathrm{NM}$ stars have proper motion membership probabilities higher than $80 \%$. However, since the mean proper motions of both IC 2488 and its surrounding field

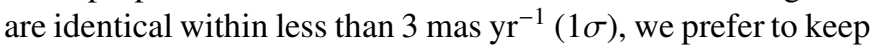
our membership assignment for these stars.

\subsection{Reddening, distance and age}

The reddening of IC 2488 was first determined from the brightest cluster members. Since probable members with $U-B<$ $-1.15 \times(B-V)+0.60$ in Fig. 4 are almost certainly earlier than $\mathrm{A} 2$, their colour excesses $E(B-V)$ and $E(U-B)$ can be calculated from the following equations (cf. Clariá 1977):

$E(B-V)=\frac{3.712 \times(B-V)-(U-B)}{3.012-0.05 \times(B-V)}$

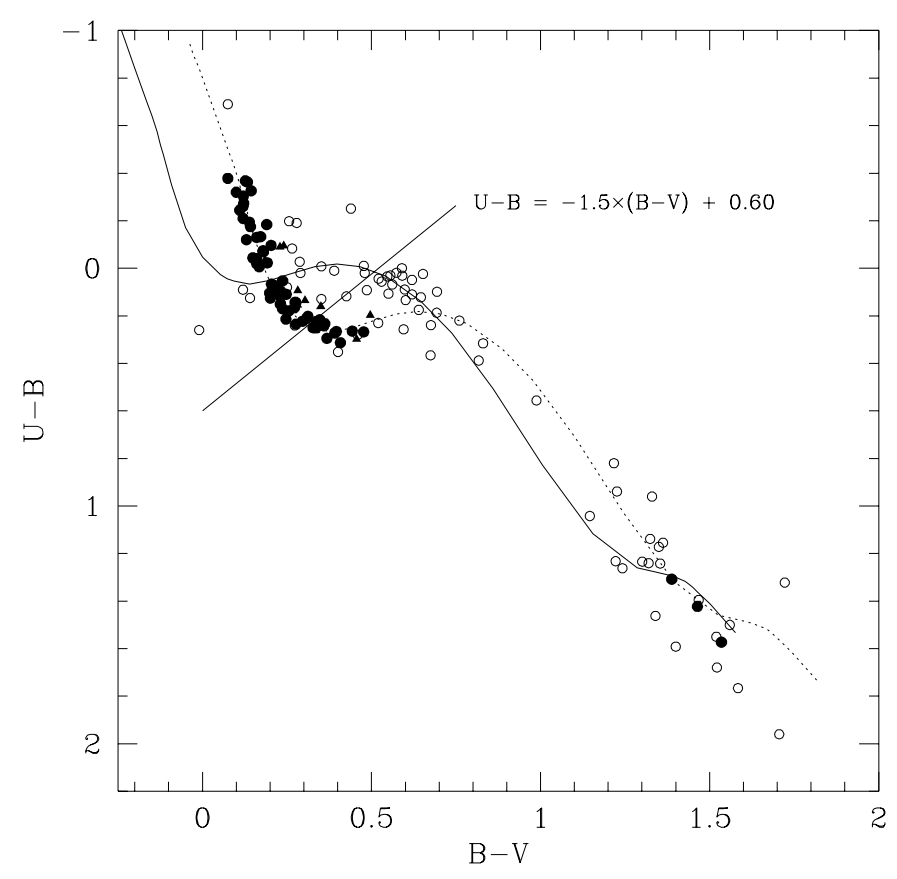

Fig. 4. $[U-B, B-V]$ colour-colour diagram for stars observed in the field of IC 2488. The Girardi et al. (2000) ZAMS for solar metallicitiy has been plotted for $E(B-V)=0.0$ (solid line) and $E(B-V)=0.24$ (dotted line). Probable members with $U-B<-1.15 \times(B-V)+0.60$ are almost certainly earlier than A2. Symbols are as in Fig. 2.

$E(U-B)=0.70 \times E(B-V)+0.05 \times E(B-V)^{2}$.

Although rigorously used, these equations should be valid only for MS stars earlier than about A2, they can also be utilized for luminosity class III and IV stars without the chance of introducing major errors. The individual colour excesses $E(B-V)$ and $E(U-B)$ are provided in Table 5. The measured full width of the observed CC diagram from these stars is $\Delta E(B-V)=$ 0.12 mag, which is slightly larger than 0.11 , the lower limit estimated by Burki (1975) for clusters with differential reddening. The mean reddening values and standard deviations from 48 stars of Table 5 are: $\langle E(B-V)\rangle=0.25 \pm 0.03$ and $\langle E(U-B)\rangle=0.17 \pm 0.02$, in very good agreement with the cluster reddening derived by P87.

Based on the assumption that we have correctly identified the cluster members, we may now estimate reddening, distance and age by fitting theoretical isochrones to the observed $\mathrm{CM}$ diagrams. Two different sets of isochrones computed with convective overshooting by the Geneva (Lejeune \& Schaerer 2001, hereafter LS01) and Padova (Girardi et al. 2000, hereafter GBBC00) groups have been used. We have first adjusted the lower cluster MS to the Zero Age Main Sequence, and then fitted the evolved portion of it using the position of the confirmed red giant members as reference, assuming solar metal content (see Sect. 4). If we had failed to consider these giant stars, isochrones covering a relatively wide age range could have fitted the upper MS adequately enough, owing to its broadness and relative verticality. However, the number of isochrones that would simultaneously match both the cluster MS and the positions of the red giants is certainly more restricted. The GBBC00's isochrone corresponding to $Z=0.019$ which best 
Table 5. Individual colour excesses for B and early A-type members of IC 2488 .

\begin{tabular}{rcc|rcc}
\hline \hline Star & $E_{(B-V)}$ & $E_{(U-B)}$ & Star & $E_{(B-V)}$ & $E_{(U-B)}$ \\
\hline 3 & 0.29 & 0.21 & 74 & 0.25 & 0.18 \\
7 & 0.21 & 0.15 & 75 & 0.29 & 0.21 \\
11 & 0.21 & 0.15 & 77 & 0.30 & 0.21 \\
12 & 0.25 & 0.18 & 78 & 0.24 & 0.17 \\
13 & 0.22 & 0.15 & 80 & 0.24 & 0.17 \\
14 & 0.29 & 0.21 & 81 & 0.25 & 0.18 \\
15 & 0.28 & 0.20 & 82 & 0.27 & 0.19 \\
17 & 0.24 & 0.17 & 86 & 0.25 & 0.18 \\
24 & 0.26 & 0.19 & 87 & 0.21 & 0.15 \\
28 & 0.23 & 0.20 & 89 & 0.32 & 0.23 \\
32 & 0.25 & 0.18 & 93 & 0.24 & 0.17 \\
33 & 0.21 & 0.15 & 96 & 0.23 & 0.16 \\
37 & 0.25 & 0.18 & 98 & 0.23 & 0.16 \\
39 & 0.24 & 0.17 & 99 & 0.20 & 0.14 \\
40 & 0.28 & 0.20 & 101 & 0.25 & 0.18 \\
41 & 0.22 & 0.15 & 102 & 0.31 & 0.22 \\
43 & 0.20 & 0.14 & 104 & 0.23 & 0.16 \\
45 & 0.21 & 0.15 & 108 & 0.24 & 0.17 \\
48 & 0.20 & 0.14 & 110 & 0.28 & 0.20 \\
49 & 0.26 & 0.19 & 111 & 0.29 & 0.21 \\
50 & 0.24 & 0.17 & 124 & 0.32 & 0.23 \\
62 & 0.29 & 0.21 & 130 & 0.20 & 0.14 \\
66 & 0.28 & 0.20 & 134 & 0.22 & 0.15 \\
69 & 0.24 & 0.17 & 143 & 0.26 & 0.19 \\
\hline
\end{tabular}

fits the cluster sequence turns out to be that for $\log t=8.25$, as shown in Figs. 2 and 3. Note that in the $(V, B-V)$ CMD (Fig. 2), this isochrone reproduces remarkably well not only the morphology of the upper MS but also the observed red giant pattern, while in the $(V, U-B) \mathrm{CMD}$ (Fig. 3) the upper cluster MS would appear to be slightly younger. This small age difference constrains the accuracy of the fitting to a confidence level higher than $80 \%$. For the sake of comparison, we have also included in Figs. 2 and 3 the GBBC00's isochrones for $\log t=8.15$ and 8.30. Figure 5 shows that the isochrone for $\log t=8.25$ calculated from the models of LS01, assuming nearly solar metal content, also compares very well with that of GBBC00. The $E(B-V)$ and $E(U-B)$ colour excesses derived from the fitting of isochrones are 0.24 and 0.17 , respectively, in very good agreement with the ones derived from B and early A-type members (see Table 5), while the apparent distance modulus turns out to be $V-M_{V}=11.20$. Assuming $R=A_{v} / E(B-V)=3.0 \pm 0.2$, the resulting total visual absorption in front of IC 2488 is then 0.72 . The true distance modulus is $V_{\mathrm{o}}-M_{v}=10.48$, which corresponds to a distance of $1250 \mathrm{pc}$ from the Sun and 96 pc below the Galactic plane. The uncertainty in $E(B-V)$ and $E(U-B)$ is about 0.04 mag and the error in the fitting to the observed sequences is estimated to be $\Delta\left(V-M_{V}\right)=0.15 \mathrm{mag}$. Taking due notice of these uncertainties, we conclude that the cluster distance may be increased or decreased by about $10 \%$. Even though the distance estimated in the present paper is nearly $13 \%$ smaller than that derived by $\mathrm{P} 87$, we believe that the present value is more reliable

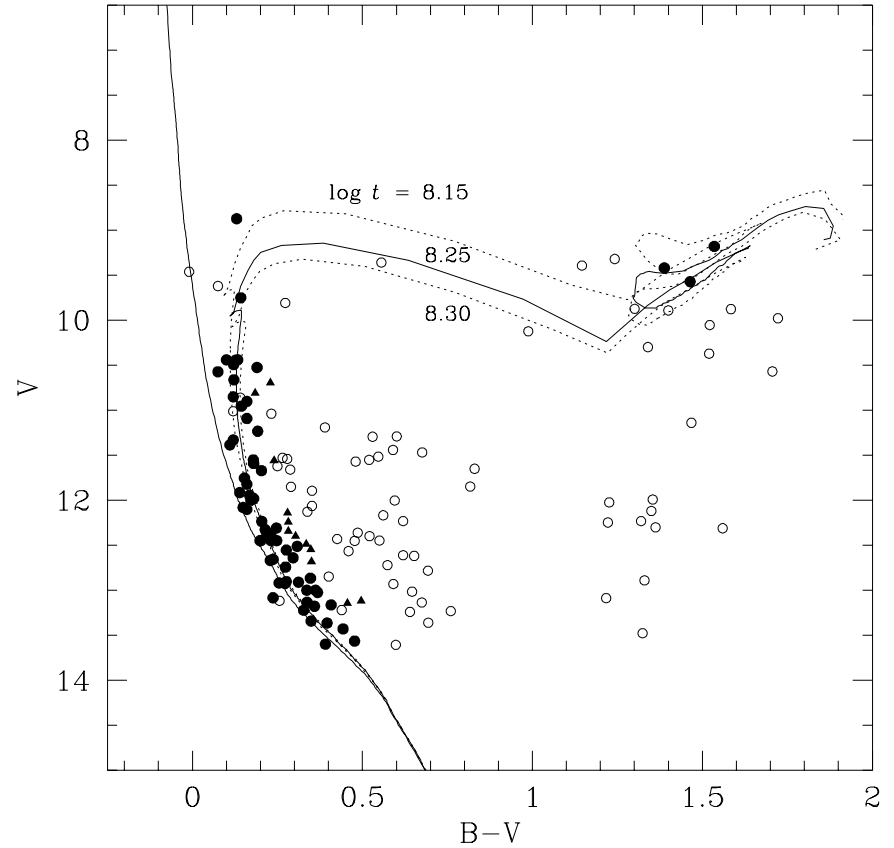

Fig. 5. The observed $[V, B-V]$ diagram for IC 2488. The theoretical isochrones computed by Lejeune \& Schaerer (2001) for $Z=$ 0.02 and $\log t=8.15,8.25$ (solid line) and 8.30, have been adjusted to $E(B-V)=0.24$ and $V-M_{V}=11.20$. Symbols are as in Fig. 2 .

because it results from the adjustment of much better defined sequences than in P87. The difference in the distances determined can also be partly accounted for by the fact that P87 does not employ red giants as the adjustment reference.

P87 speculated about the possibility that the planetary nebula ESO 166-PN21, also known as RCW 44 and Wr 17-31 and catalogued as PN 277.7-3.5 in Acker et al. (1992), could be related to IC 2488. A distance of $(1.2 \pm 0.2) \mathrm{kpc}$, compatible with the one here obtained for IC 2488 , has been derived by Peña et al. (1997) for the nebula. However, if we adopt the coordinates given by Lyngå (1987) and Acker et al. (1992) for the cluster centre and nebula, respectively, then ESO 166-PN21 lies north-east more than four radii away from the cluster centre. We thus believe that the probability of IC 2488 being related to the planetary nebula is very low.

\section{Red stars in the cluster field}

\subsection{Cluster membership and interstellar reddening}

Among the stars observed in the $U B V$ system, fourteen stars, most of which were observed in the $D D O$ and Washington systems (Tables 2 and 3), could be yellow or red giant members according to their positions in the CM diagrams.

Based on the Coravel radial-velocity data (Table 4), only stars \#97, 115 and 126 can be treated as indisputable members of IC 2488, since their radial velocities are in very close agreement. The mean radial velocity of these stars - one of them being a spectroscopic binary $(\mathrm{SB})-$ is $(-2.63 \pm 0.06) \mathrm{km} \mathrm{s}^{-1}$ (s.e. of the mean) and has been adopted for the cluster. We note that the membership probabilities assigned to these stars by Dias et al. (2001) from their proper motions are higher than $80 \%$ 
Table 6. Red giant membership results.

\begin{tabular}{rccccccccc}
\hline \hline Star & $E(B-V)_{\mathrm{GK}}$ & $\sigma_{E}$ & LC & \multicolumn{2}{c}{ MK } & \multicolumn{5}{c}{ Membership } \\
& & & Pred. & DDO & \multicolumn{2}{c}{ Photom. } & $V_{\mathrm{r}}$ & $\operatorname{Pr}(\%)$ & Fin. \\
& & & & & (A) & $(\mathrm{B})$ & & & \\
\hline 1 & 0.07 & 0.06 & II-III & K3/4III-IV & $\mathrm{nm}$ & $\mathrm{pm}$ & $\mathrm{nm}$ & - & $\mathrm{nm}$ \\
2 & 0.08 & 0.05 & II & K3/4III & $\mathrm{nm}$ & $\mathrm{pm}$ & $\mathrm{nm}$ & - & $\mathrm{nm}$ \\
30 & 0.23 & 0.04 & II & G8/9II & $\mathrm{m}$ & $\mathrm{m}$ & $\mathrm{nm}$ & 92 & $\mathrm{pm}$ \\
35 & 0.07 & 0.04 & II & K1III & $\mathrm{nm}$ & $\mathrm{pm}$ & $\mathrm{nm}$ & 0 & $\mathrm{~nm}$ \\
$67^{a}$ & - & - & - & - & - & - & $\mathrm{nm}$ & - & $\mathrm{nm}$ \\
68 & 0.19 & 0.02 & II & K4III & $\mathrm{nm}$ & $\mathrm{pm}$ & $\mathrm{nm}$ & - & $\mathrm{nm}$ \\
97 & 0.29 & 0.05 & II & K0II & $\mathrm{m}$ & $\mathrm{m}$ & $\mathrm{m}$ & 81 & $\mathrm{~m}$ \\
115 & 0.30 & 0.06 & II & K2II & $\mathrm{m}$ & $\mathrm{m}$ & $\mathrm{m}$ & 93 & $\mathrm{~m}$ \\
126 & 0.27 & 0.06 & II & K1/2II & $\mathrm{m}$ & $\mathrm{m}$ & $\mathrm{m}$ & 83 & $\mathrm{~m}$ \\
129 & 0.15 & 0.03 & II & K3/4III & $\mathrm{nm}$ & $\mathrm{pm}$ & $\mathrm{nm}$ & 27 & $\mathrm{~nm}$ \\
144 & $>0.7$ & - & II & - & $\mathrm{nm}$ & & $\mathrm{nm}$ & - & $\mathrm{nm}$ \\
145 & 0.12 & 0.06 & II & K2III-IV & $\mathrm{nm}$ & $\mathrm{nm}$ & $\mathrm{nm}$ & - & $\mathrm{nm}$ \\
\hline
\end{tabular}

${ }^{a}$ Single-lined spectroscopic binary with orbit determined by Mermilliod et al. (1997). DDO colours outside the range of Janes's (1977) calibration.

(Col. 9 of Table 6). Star \#67 (HDE 300105) is a single-lined SB whose orbit has been determined by Mermilliod et al. (1997). Its systemic velocity differs by about $17 \mathrm{~km} \mathrm{~s}^{-1}$ from the cluster mean velocity, so this star is undoubtedly a non-member of IC 2488 .

Cluster membership among the possible red giants was also examined by applying two photometric criteria - denoted (A) and (B) - described by Clariá \& Lapasset (1983), which are based on combined $B V$ and $D D O$ data. Criterion (A) requires computing for each $\mathrm{G}$ or K-type star the difference $\Delta E$ (in absolute value) between $E(B-V)_{\mathrm{GK}}$ and $E(B-V)_{\mathrm{MS}}$, the latter value being the colour excess obtained from the MS stars. No differential reddening being present, a red star is considered to be a member $(\mathrm{m})$, probable member $(\mathrm{pm})$, or non-member $(\mathrm{nm})$, if the former difference is found to be: $\Delta E \leq \sigma_{E}$ (standard deviation of $\left.E(B-V)_{\mathrm{GK}}\right), \sigma_{E}<\Delta E \leq 2 \sigma_{E}$, or $\Delta E>2 \sigma_{E}$, respectively. On the other hand, criterion (B) is based on the agreement between the predicted luminosity class (LC) which a given star would have in order to be a cluster member, and that derived from the $D D O$ indices. Criterion (B) is then applied in such a way that a red star is considered to be a member, probable member, or non-member, if the difference $\Delta L$ (in absolute value) between the predicted LC and that derived from the DDO colours is found to be: $\Delta L \leq 0.5 \mathrm{LC}, 0.5 \mathrm{LC}<\Delta L \leq$ $1 \mathrm{LC}$, or $\Delta L>1 \mathrm{LC}$, respectively.

In order to apply the above photometric criteria, we used the cluster reddening and distance modulus derived in Sect. 3. The $D D O$ colours were dereddened according to the reddenning coefficients of McClure (1973) and the predicted LC for each star was determined from Schmidt-Kaler's (1982) calibration assuming $R=A_{v} / E(B-V)=3.0$. Columns (2)-(10) of Table 6 display in succession the colour excess $E(B-V)_{\mathrm{GK}}$ derived from Janes's (1977) method, the standard deviation $\sigma_{E}$ computed using Eq. (2) of Clariá \& Lapasset (1983), the LC the star would have in order to be a cluster member, the MK spectral type derived from the $D D O$ colours using the calibration of Clariá et al. (1994), the results from applying the photometric and kinematic criteria, and the membership status finally adopted. We used the individual values of $\sigma_{(B-V)}$ for each star to compute $\sigma_{E}$. Star \#67 (HDE 300105), a known spectroscopic binary (Mermilliod et al. 1997), falls outside the range of Janes's (1977) calibration. The $E(B-V)_{\mathrm{GK}}$ values of the three Coravel giant members average to $\langle E(B-V)\rangle=$ $0.29 \pm 0.03$, a value somewhat larger than those derived in Sect. 3.2. Individual $E(B-V)_{\mathrm{GK}}$ values listed in Table 6 were used to correct the $D D O$ colours for interstellar reddening.

According to the above criteria, only stars \#30, 97, 115 and 126 have a high probability of being cluster giants, the remaining stars being very likely field stars. Even if the spectral types derived from $D D O$ photometry are simply estimates, it is encouraging to see that all likely members are estimated to have luminosity class II, while all non-members are estimated to have lower luminosity. Three of the four photometric members are also Coravel members. Therefore, as shown in previous papers (see, e.g., Clariá et al. 1999), once again $B V-D D O$ photometric membership probabilities appear to be in very good agreement with those obtained from Coravel radial velocities. The only conflictive case in the present study appears to be star \#30 (HDE 302174), a Coravel non member, which instead appears to be cluster member according to the photometric criteria (A) and (B) as well as to the Dias et al. (2001) absolute proper motions. The radial velocity of this star differs considerably from that of the cluster mean and although the possibility of this star being a long-period binary system still remains, we would be inclined to consider it as a probable non-member.

\subsection{Metal abundance}

\subsubsection{DDO analysis}

It is well known that the $C(41-42)$ index of the $D D O$ photometry measures the strength of the $\lambda 4216$ band of cyanogen of $G$ and $\mathrm{K}$ stars, so that the larger the index the greater the absorption by the band. Janes (1975) has shown that the cyanogen 
Table 7. Abundance parameters of red stars in the cluster field.

\begin{tabular}{rrrrrrr}
\hline \hline Star & $\Delta \mathrm{CN}$ & $\Delta_{1}^{\prime}$ & $\Delta_{2}^{\prime}$ & $\Delta_{3}^{\prime}$ & $\Delta_{4}^{\prime}$ & $\Delta_{5}^{\prime}$ \\
\hline 1 & 0.018 & -0.061 & 0.004 & -0.058 & -0.070 & -0.071 \\
2 & -0.011 & -0.039 & -0.002 & -0.041 & -0.038 & -0.040 \\
30 & 0.070 & 0.017 & -0.003 & 0.016 & 0.014 & 0.012 \\
35 & -0.026 & -0.137 & -0.034 & -0.171 & -0.103 & -0.117 \\
68 & 0.010 & -0.037 & -0.024 & -0.055 & -0.027 & -0.049 \\
97 & 0.084 & 0.061 & 0.002 & 0.083 & 0.057 & 0.056 \\
115 & 0.092 & 0.045 & -0.004 & 0.042 & 0.033 & 0.030 \\
126 & 0.033 & 0.006 & -0.010 & -0.004 & 0.004 & -0.001 \\
129 & -0.037 & -0.084 & -0.010 & -0.095 & -0.076 & -0.084 \\
145 & 0.062 & -0.099 & -0.034 & -0.134 & -0.066 & -0.079 \\
\hline
\end{tabular}

anomaly $\delta \mathrm{CN}$ - defined as the excess (positive $\delta \mathrm{CN}$ ) or deficiency of the $\mathrm{CN}$ index $C_{\mathrm{o}}(41-42)$ in magnitudes over the standard value for a star of the same $C_{\mathrm{o}}(45-48)$ and $C_{\mathrm{o}}(42-45)$ indices - is well correlated with $[\mathrm{Fe} / \mathrm{H}]$. However, some inconsistencies in the definition and calculation of $\delta \mathrm{CN}$ pointed out by Piatti et al. (1993, hereafter PCM93) led them to redefine the cyanogen anomaly - now denoted $\triangle \mathrm{CN}$ - as the difference between the $C_{\mathrm{o}}(41-42)$ index and the standard value of this index corresponding to a star with the same temperature and surface gravity [and not with the same $C_{\mathrm{o}}(45-48)$ and $C_{\mathrm{o}}(42-45)$ indices] as the star in question.

Column (2) of Table 7 lists the $\triangle \mathrm{CN}$ values calculated as described in PCM93. The mean cyanogen anomaly for the three confirmed red giant members is $\langle\Delta \mathrm{CN}\rangle=0.070 \pm$ 0.015 (s.e of the mean). This implies $[\mathrm{Fe} / \mathrm{H}]=0.10 \pm 0.06$, if Eq. (8) of PCM93 is used. We note that if the old cyanogen anomaly $\delta \mathrm{CN}$ instead of $\Delta \mathrm{CN}$ had been used, then the resulting iron-to-hydrogen ratio would have been scarcely 0.1 dex lower. We thus conclude from the $D D O$ data that IC 2488 is slightly more metal-rich than the Sun.

\subsubsection{Washington abundance parameters}

As shown by Geisler et al. (1991, hereafter GCM91), their metal abundance calibration for the Washington system presents calibrations for five combinations of the various abundance and temperature indices. To determine the cluster metallicity we have adopted their recommended procedure and calibration. Specifically, we used the reddening-corrected isoabundance relations between $(C-M)_{\mathrm{o}}$ and $\left(T_{1}-T_{2}\right)_{\mathrm{o}},\left(M-T_{1}\right)_{\mathrm{o}}$ and $\left(T_{1}-T_{2}\right)_{\mathrm{o}},\left(C-T_{1}\right)_{\mathrm{o}}$ and $\left(T_{1}-T_{2}\right)_{\mathrm{o}},(C-M)_{\mathrm{o}}$ and $\left(M-T_{2}\right)_{\mathrm{o}}$ and $\left(C-T_{1}\right)_{0}$ and $\left(M-T_{2}\right)_{0}$, where the first index is primarily metallicity sensitive and the second, primarily temperature sensitive.

For each observed star we determined five metallicity indices $\Delta_{1}^{\prime}-\Delta_{5}^{\prime}$, as defined in GCM91. For this purpose, we used the reddening ratios given by GCM91 and the $E(B-V)_{\mathrm{GK}}$ values of Table 6 . Stars \#113 and 144 were omitted because they fall outside the range of the abundance calibrations. Figure 6 displays the five unreddened Washington CC diagrams for the stars observed in IC 2488. Iso-abundance relations for $[\mathrm{Fe} / \mathrm{H}]=+0.5,0.0,-0.5$ and -1.0 are shown in

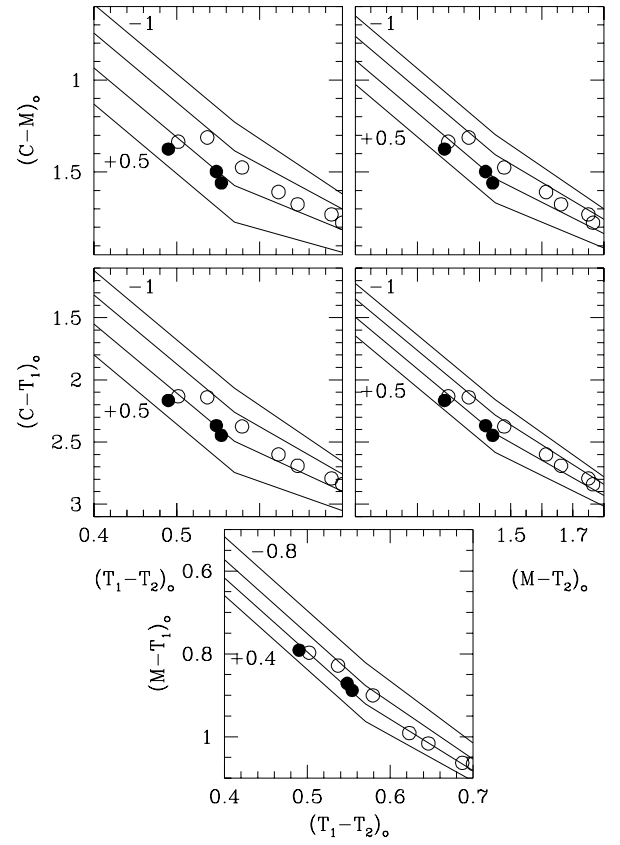

Fig. 6. The red giant candidates observed in the Washington system plotted in the different unreddened two-colour diagrams from which the $\Delta_{i}^{\prime}$ abundance parameters are derived. Iso-abundance relations from Geisler et al. (1991) for 0.5 dex intervals from $[\mathrm{Fe} / \mathrm{H}]=-1.0$ to +0.5 are shown, except for the $\left(M-T_{1}\right)_{\mathrm{o}}$ vs. $\left(T_{1}-T_{2}\right)_{\mathrm{o}}$ diagram wherein iso-abundance relations for 0.4 dex intervals from $[\mathrm{Fe} / \mathrm{H}]=$ -0.8 to +0.4 are given. Stars confirmed as red giant members from Coravel, proper motions and photometric criteria are represented with filled circles, while the only possible member is represented by a filled triangle.

Fig. 6, except for the $\left(M-T_{1}\right)_{\mathrm{o}}$ versus $\left(T_{1}-T_{2}\right)_{\mathrm{o}}$ diagram wherein iso-abundance lines corresponding to $[\mathrm{Fe} / \mathrm{H}]=+0.4$, $0.0,-0.4$ and -0.8 are plotted. Star \#46, not observed in the $D D O$ system, and the spectroscopic binary HD 300105 (star \#67) with unknown reddening, have not been plotted in Fig. 6. It is worth underlining that the three confirmed giant members (stars \#97, 115 and 126) lie very close to the $[\mathrm{Fe} / \mathrm{H}]=0.0$ iso-abundance lines in Fig. 6 , thereby confirming the results obtained from the $D D O$ photometry. The five Washington abundance indices are given in Cols. (3)-(7) of Table 7. The resulting mean values and standard deviations of the mean from three cluster giants are:

$<\Delta_{1}^{\prime}>=<\Delta^{\prime}(C-M)_{T_{1}-T_{2}}>=0.037 \pm 0.013$

$<\Delta_{2}^{\prime}>=<\Delta^{\prime}\left(M-T_{1}\right)_{T_{1}-T_{2}}>=-0.004 \pm 0.003$,

$\left.<\Delta_{3}^{\prime}\right\rangle=\left\langle\Delta^{\prime}\left(C-T_{1}\right)_{T_{1}-T_{2}}>=0.040 \pm 0.021\right.$,

$<\Delta_{4}^{\prime}>=<\Delta^{\prime}(C-M)_{M-T_{2}}>=0.043 \pm 0.006$

$<\Delta_{5}^{\prime}>=<\Delta^{\prime}\left(C-T_{1}\right)_{M-T_{2}}>=0.028 \pm 0.013$,

which imply $[\mathrm{Fe} / \mathrm{H}]_{1}=0.11,[\mathrm{Fe} / \mathrm{H}]_{2}=-0.05,[\mathrm{Fe} / \mathrm{H}]_{3}=$ $0.10,[\mathrm{Fe} / \mathrm{H}]_{4}=0.19$, and $[\mathrm{Fe} / \mathrm{H}]_{5}=0.12$, if the calibrations of GCM91 are used. Since the difference between the Washington abundances derived from the iron lines and those obtained from the blue spectral features contaminated by $\mathrm{CN}$ and $\mathrm{CH}$ is not overly significant in terms of the photometric and calibration errors, we conclude that the cluster giants are not enriched in elements of the $\mathrm{CNO}$ group. The unweighted mean 
of the five Washington abundance estimates, i.e., $[\mathrm{Fe} / \mathrm{H}]=$ $0.09 \pm 0.08$, is in very good agreement with the $D D O$ metal content.

\section{Summary and conclusions}

The present photometric and kinematic study of IC 2488 supersedes the previous photometric study by P87 on account of the larger number of stars observed in the cluster field. The following are the main conclusions:

(i) The $U B V \mathrm{CM}$ and $\mathrm{CC}$ diagrams reveal some $\mathrm{B}$ and several A-type stars not previously identified by P87. The analysis of the new photometric data warrants our claim that 63 stars are probable members, one of which is a blue straggler (CD-56 $\left.6^{\circ} 2787\right)$, while 13 stars are possible members. Indeed, further observation of the former, e.g. spectroscopy, would probably be instrumental in confirming its membership status.

(ii) The full width of the observed cluster MS in the CC diagram measured from $B$ and early A-type stars, is compatible with the cluster reddening being uniform, the mean $E(B-V)$ value being $0.24 \mathrm{mag}$. An apparent distance modulus $V-M_{V}=$ $11.20 \pm 0.15$, equivalent to a distance of $(1250 \pm 120) \mathrm{pc}$, is derived for IC 2488 . This distance is nearly $13 \%$ smaller than that derived by P87.

(iii) Accurate radial velocities of 13 red giant candidates confirm cluster membership for three stars, one of them being a new spectroscopic binary. These stars also appear to be cluster members, a result based on two independent photometric criteria and proper motion membership probabilities. The mean radial velocity from 3 giant members is $(-2.63 \pm 0.06) \mathrm{km} \mathrm{s}^{-1}$.

(iv) The planetary nebula ESO 166-PN21, a probable cluster member according to P87, lies north-east more than four cluster radii away from the cluster centre. Therefore, although it is located at the same distance as IC 2488, the chance of ESO 166-PN21 being related to the cluster is considerably low.

(v) IC 2488 has a mean cyanogen anomaly $\langle\Delta \mathrm{CN}\rangle=$ $0.070 \pm 0.011$, which implies $[\mathrm{Fe} / \mathrm{H}]=0.10 \pm 0.06$. This value is in excellent agreement with the one derived from five independent Washington abundance indicators $([\mathrm{Fe} / \mathrm{H}]=0.09 \pm$ 0.08 ). Adopting a metal content $Z=0.019$, the isochrones for $\log t=8.25(180 \mathrm{Myr})$ computed from the models of Girardi et al. (2000) and Lejeune \& Schaerer (2001), reproduce quite effectively not only the upper main sequence but also the observed red giant pattern, particularly in the $(V, B-V)$ CMD.

Acknowledgements. We have used an image from the Digitized Sky Survey, produced at the Space Telescope Science Institute under U.S. Government grant NAG W-2166. We would like to thank the CTIO and Las Campanas staff members and night assistants for their kind hospitality and support during the observing runs. We also thank the referee, Dr. I. Negueruela, for his invaluable suggestions, which have allowed us to improve the manuscript. The assistance of Mrs. M.A. Bosio in the data reduction and Mrs. A.V. Ahumada in the preparation of some figures is also acknowledged. JJC, AEP and EL gratefully acknowledge financial support from the Argentinian institutions AGENCIA NACIONAL DE PROMOCIÓN CIENTIFICA
Y TECNOLÓGICA (ANPCyT), CONICET, SECYT (UNC) and AGENCIA CÓRDOBA CIENCIA. The radial-velocity programme has been supported by continuous grants from the Swiss National Foundation for Scientific Research (FNRS).

\section{References}

Acker, A., Marcout, J., Ochsenbein, F., Stenholm, B., \& Tylenda, R. 1992, The Strasbourg-ESO Catalogue of Galactic Planetary Nebulae

Baranne, A., Mayor, M., \& Poncet, J.-L. 1979, Vistas in Astron., 23, 279

Baranne, A., Queloz, D., Mayor, M., et al. 1996, A\&AS, 119, 373

Baumgardt, H., Dettbarn, C., \& Wielen, R. 2000, A\&AS, 146, 251

Burki, G. 1975, A\&A, 43, 37

Canterna, R. 1976, AJ, 81, 228

Clariá, J. J. 1977, A\&AS, 27, 145

Clariá, J. J., \& Lapasset, E. 1983, JAp\&A, 4, 117

Clariá, J. J., \& Lapasset, E. 1985, MNRAS, 214, 229

Clariá, J. J., Lapasset, E., \& Bosio, M. A. 1991, MNRAS, 249, 193

Clariá, J. J., Mermilliod, J.-C., \& Piatti, A. E. 1999, A\&AS, 134, 301

Clariá, J. J., Piatti, A. E., \& Lapasset, E. 1994, PASP, 106, 436

Clariá, J. J., Piatti, A. E., \& Lapasset, E. 1998, A\&AS, 128, 131

Cousins, A. W. J. 1973, Mem. R. Astron. Soc., 77, 22

Cousins, A. W. J. 1974, Mon. Notes Astron. Soc. S. Afr., 33, 149

Dean, D. F. 1981, South Africa Astron. Obs. Cirs., 6, 10

Dias, W. S., Lépine, J. R. D., \& Alessi, B. S. 2001, A\&A, 376, 441

Geisler, D., Clariá, J. J., \& Minniti, D. 1991, AJ, 102, 1836 (GCM91)

Girardi, L., Bressan, A., Bertelli, G., \& Chiosi, C. 2000, A\&AS, 141, 371 (GBBC00)

Glushkova, E. V., Zabolotskikh, M. V., Rastorguev, A. S., et al. 1997, PAZh, 23, 90

Graham, J. A. 1982, PASP, 94, 244

Harris, H. C., \& Canterna, R. 1979, AJ, 84, 1750

Janes, K. A. 1975, ApJS, 29, 161

Janes, K. A. 1977, PAPS, 89, 576

Landolt, A. U. 1973, AJ, 78, 959

Lejeune, T., \& Schaerer, D. 2001, A\&A, 366, 538 (LS01)

Lyngå, G. 1987, Catalogue of Open Cluster Data, Strasbourg: Centre de Données Stellaires

McClure, R. D. 1973, Spectral Classification and Multicolour Photometry (D. Reidel Publishing Co., Dordrecth, Holland), p. 162

McClure, R. D. 1976, AJ, 81, 182

Mermilliod, J.-C., Andersen, J., \& Mayor, M. 1997, A\&A, 319, 481

Piatti, A. E., Clariá, J. J., \& Minniti, D. 1993, JAp\&A, 14, 145 (PCM93)

Pedreros, M. 1987, AJ, 94, 92 (P87)

Peña, M., Ruiz, M. T., Bergeron, P., et al. 1997, A\&A, 317, 911

Schmidt-Kaler, Th. 1982, in Landolt-Bornstein, Numerical Data and Functional Relationships in Science and Technology, New Series, group VI, Vol. 2b, ed. K. Schaifers, \& H. H. Voigt (Berlin, Springer Verlag)

Ruprecht, J. 1966, Bull. Inst. Czech., 17, 34

Sanders, W. L. 1971, A\&A, 14, 226

Trumpler, R. J. 1930, Lick Obs. Bull., 14, 154

Udry, S., Mayor, M., \& Queloz, D. 1999, in Precise Stellar Radial Velocities, IAU Colloq. 170, ed. J. B. Hearnshaw, \& C. D. Scarfe, ASP Conf. Ser., 185, 367 